
\title{
25 Research Square \\ Chiral Photochemistry of Achiral Molecules: The Emblematic Case of Stilbene and Stiff-Stilbene
}

\author{
Umberto Raucci
}

Italian Institute of Technology

Hayley Weir

Stanford University

Christoph Bannwarth

Stanford University https://orcid.org/0000-0003-3242-496X

\section{David Sanchez}

Lawrence Livermore National Laboratory https://orcid.org/0000-0003-0697-7579

Todd Martinez ( $\nabla$ toddjmartinez@gmail.com )

Stanford University https://orcid.org/0000-0002-4798-8947

\section{Article}

Keywords: chirality, potential energy surfaces, electronically prochiral molecules

Posted Date: October 5th, 2021

DOl: https://doi.org/10.21203/rs.3.rs-955011/v1

License: (c) (1) This work is licensed under a Creative Commons Attribution 4.0 International License. Read Full License

Version of Record: A version of this preprint was published at Nature Communications on April 19th, 2022. See the published version at https://doi.org/10.1038/s41467-022-29662-1. 


\section{Abstract}

Chirality is a fundamental molecular property governed by the topography of the potential energy surface (PES). The barrier height between two enantiomers relative to the thermal energy dictates the timescale for their interconversion (and thus whether this can be observed). Thermally achiral molecules interconvert rapidly when the interconversion barrier is comparable to or lower than the thermal energy, in contrast to thermally stable chiral configurations. In principle, a change in the PES topography on the excited electronic state may diminish interconversion, leading to electronically prochiral molecules that can be converted from achiral to chiral by electronic excitation. We demonstrate that this is the case for two prototypical examples - cis-stilbene and cis-stiff stilbene. Both systems exhibit unidirectional photoisomerization for each enantiomer as a result of their electronic prochirality. We propose and simulate an experiment to demonstrate this effect in cis-stilbene based on its interaction with circularly polarized light. Our results highlight the drastic change in chiral behavior upon electronic excitation, opening up the possibility for asymmetric photochemistry from an effectively nonchiral starting point.

\section{Background}

Chirality is a symmetry property widespread in nature, and examples include the molecules of life (e.g. proteins, nucleic acids). A molecule is chiral when it cannot be superimposed on its mirror image (enantiomer), and chirality occurs in asymmetric or dissymmetric systems (i.e. molecules that do not have any symmetry elements or have only rotation axes). The effective (i.e., observable) chirality of a given molecule is governed by the topography of its potential energy surface (PES), specifically the height of the barrier separating the minima of the two enantiomers (the PES is symmetric respect to an interconversion coordinate, Fig. 1). If the interconversion barrier is much higher than the thermal energy $\left(k_{B} T\right)$, the two enantiomers can be isolated and easily characterized (i.e., thermally stable chiral molecules, Fig. 1a). Otherwise, rapid interconversion between the two enantiomeric configurations takes place making them inseparable (i.e., thermally achiral molecules, Fig. 1a).

By tuning the PES topography it is possible to influence and control the inherent chiral behavior of the molecule. One such way is by promoting the molecule to an electronic excited state where the new electronic configuration can induce an increase/decrease of the interconversion barrier resulting in a shift of the enantiomeric ratio. Furthermore, the electronic excitation can introduce other accessible photoreactions (e.g. photoisomerization) which quench enantiomeric interconversion (Fig. 1b). In this case, the molecule can be considered electronically prochiral: it can be converted from achiral to chiral by electronic excitation.

Herein, we examine the concept of electronic prochirality via the relationship between enantiomer interconversion and cis-trans photoisomerization around a carbon-carbon double bond. In the recent decades, this has been exploited to generate a local asymmetry in the PES resulting in unidirectional motion. Indeed, the preferential selectivity of a particular direction of motion is the result of a nonequilibrium process in which the local topography of the PES is either sloped or characterized by a 
substantially lower energy barrier in that direction when compared to other possible pathways. The presence of chiral elements represents the simplest way to break the symmetry of the PES on the atomistic scale, ensuring that one rotational direction, e.g. clockwise (CW) or anticlockwise (ACW), is energetically preferred over the other.

Inspired by this principle, several generations of artificial light-driven molecular motors have been reported..$^{1-9}$ In these synthetic nanomachines, the introduction of asymmetric elements (e.g. stereogenic center or chirality axis) results in unidirectional rotation (CW or ACW) around a carbon-carbon double bond. Feringa and co-workers achieved full $360^{\circ}$ unidirectional rotation by designing stericallyovercrowded alkenes. ${ }^{2,4,5}$ Here, a carbon-carbon double bond connects the stator and the rotor, and axial chirality is enforced by steric hindrance between the two halves. Bulky substituents in strategic positions around the rotational axis induce large twisting of the double bond, directing the olefinic bond rotation during the light-induced isomerization. ${ }^{2,5}$ Marchand and co-workers reported that without axial chirality a single stereocenter in the allylic position of the isomerizing double bond is enough to ensure significant unidirectionality in the photoisomerization. ${ }^{6}$ Recently, Wang and et al. exploited the axial chirality of cyclohexenylidene group to achieve unidirectional motion in a protonated Schiff-base. ${ }^{7}$

Here, we extend these findings to simple, highly symmetric molecules that are thermally achiral in the electronic ground state, where rapid interconversion between the two enantiomeric configurations takes place. Our simulations suggest that for electronically prochiral molecules, chirality can be enhanced or "stiffened" when the molecule is promoted to the excited state despite thermal achirality in the ground state. We demonstrate this effect on the cis-trans photoisomerization of 1,2-diphenylethylene (stilbene) and 1-1'-bis-indanylidene (stiff-stilbene).

\section{Results}

Stilbene and stiff-stilbene represent prototypical examples for carbon-carbon double bond photoisomerization (Fig. 2). The steric hindrance between the phenyl groups prevents cis-stilbene and cisstiff-stilbene from being completely planar in the ground electronic state $\left(\mathrm{S}_{0}\right)$, resulting in a reduction in symmetry from $\mathrm{C}_{2 v}$ to $\mathrm{C}_{2}$, with the $\mathrm{C}_{2}$ rotational axis perpendicular to the ethylene bond. The $\mathrm{C}_{2}$ axis represents the chirality axis for the molecule, and two helically twisted enantiomers arise for both cisstilbene and cis-stiff-stilbene showing right-hand and left-hand axial chirality ( $P$ and $M$ respectively, Fig. 2).

Although the photoisomerization of stilbene and stiff-stilbene has been the topic of numerous theoretical and experimental studies over the past century, ${ }^{10-15}$ to our knowledge the cis-trans photoisomerization dynamics has never been explored from the perspective of its rotational directionality. Here, we explore the rotational directionality in the non-adiabatic dynamics of the $P$ and $M$ enantiomers of cis-stilbene and cis-stiff-stilbene in the gas-phase using graphical processing unit (GPU)-accelerated multiconfigurational

electronic structure theory in TeraChem ${ }^{16-18}$ coupled to ab initio multiple spawning (AIMS). ${ }^{19-21}$ 
To simulate the unidirectional photoisomerization in both cis-stilbene and cis-stiff-stilbene, AIMS trajectories for both $P$ and $M$ enantiomers are initiated from thirty initial conditions (positions and momenta) sampled from a $\mathrm{OK}$ harmonic Wigner distribution around their respective ground state minimum. Fig. 3 shows the time-evolution of the central ethylenic twist angle ( $\tau$ in Fig. 2 ) around the photoisomerizing carbon-carbon bond for the two enantiomers ( $P$ and $M)$ of cis-stilbene and cis-stiffstilbene on the first singlet excited state PES, $S_{1}$. We observe that the photoisomerization dynamics for each isomer are unidirectional: the evolution of the $P$ enantiomer shows an increasing value of the $\tau$ angle until the $P \mathrm{~S}_{1} / \mathrm{S}_{0}$ conical intersection (Cl) region is reached at $90^{\circ}$ (CW rotation), whereas the excitation of the $M$ isomer leads to ACW rotation of $t$ to $-90^{\circ}$ at the $M S_{1} / S_{0} \mathrm{Cl}$.

In addition to the photoisomerization around $\mathrm{t}$, cis-stilbene may also relax back to the ground state via photocyclization leading to $4 \mathrm{a}, 4 \mathrm{~b}$-dihydrophenanthrene (DHP). As a consequence of the unidirectional dynamics, an enantioselective photocyclization is observed. Indeed, the $\mathrm{CW}$ rotation in $(P)$-cis-stilbene leads to $(4 \mathrm{a} S, 4 \mathrm{~b} S)$-4a,4b-dihydrophenanthrene ( $S, S$ DHP), while (4aR,4bR)-4a,4b-dihydrophenanthrene $(R, R-\mathrm{DHP})$ is formed from the ACW motion in (M)-cis-stilbene.

No $P$-M helical inversion is observed on $\mathrm{S}_{1}$ for both cis-stilbene and cis-stiff-stilbene indicating the occurrence of excited state axial chirality that leads to unidirectional photoisomerization. Hence, in spite of being formally achiral at thermal equilibrium on $\mathrm{S}_{0}$ due to the fast helical inversion process (inversion barriers are 1.6 and $4.9 \mathrm{kcal} / \mathrm{mol}$ for stilbene and stiff-stilbene, respectively), cis-stilbene and cis-stiffstilbene behave as chiral molecules during their short life on $\mathrm{S}_{1}$. This behavior can be easily rationalized by inspecting the frontier molecular orbitals involved in the electronic excitation (Fig. S1). Indeed, the $\mathrm{S}_{0}{ }^{\circledR} \mathrm{S}_{1}$ transition corresponds to a HOMO ${ }^{\circledR}$ LUMO p-p* one electron excitation characterized by a transfer of electron density from the central ethylenic bond to the $p$ orbitals of the adjacent carbon pairs. The new electronic arrangement reached on $\mathrm{S}_{1}$ lowers the barrier for the rotation around the central carbon-carbon double bond and hinders the rotation of the phenyl groups necessary for the $P-M$ inversion. As a result, unidirectional photoisomerization is observed.

Nevertheless, in order to achieve photo-induced net unidirectional motion, the preferential excitation of a specific enantiomer is required. Photoexcitation with nonpolarized or linearly polarized light is unsuitable for this purpose because it would lead to a racemate on the excited state. In contrast, the differential absorption of circularly polarized light $(\mathrm{CPL})$ results in bands with opposite sign in the electronic circular dichroism (ECD) spectrum for both enantiomers, meaning that an excess of one enantiomer is excited over the other. In Fig. S2, we present the simulated relative absorption spectra for right circularly polarized light (r-CPL) of the $(M)$ - and $(P)$-conformers of cis-stilbene. We use the quantum mechanically determined optical anisotropy Kuhn factor, $g$, (ratio of the dipole strength and the rotatory strength) ${ }^{22}$ for the $S_{0}{ }^{\circledR}$ $\mathrm{S}_{1}$ excitation to evaluate the enantiomeric excess of excited chiral species following r-CPL absorption (a more detailed discussion is reported in the $\mathrm{SI}$ ). In the optical window 230-350 nm, the $M$ enantiomer preferentially absorbs the $\mathrm{r}-\mathrm{CPL}$, while the $\mathrm{I}-\mathrm{CPL}$ is preferentially absorbed by the $P$ conformer. As a consequence, the excitation of the ground state sample of cis-stilbene with r-CPL will preferentially excite 
the left-handed helical twisted $M$-enantiomer, whereas the $P$ enantiomer can be preferentially excited with I-CPL.

To supplement our findings for the unidirectional photoisomerization of cis-stilbene, we simulated a rather straightforward experiment based on the enantioselective photocyclization leading to S,SDHP and $R, R$-DHP. Indeed, the excitation of cis-stilbene (existing as a racemic mixture of the $P$ and $M$ enantiomers at thermal equilibrium) with non-polarized UV light will lead to a racemic mixture of the chiral DHP photoproduct, whereas an excess of one enantiomer over the other is expected with the CPL excitation. Due to the fact that DHP can absorb CPL in the same optical window as cis-stilbene, the enantiomeric excess (ee) of DHP reached at the photo-stationary state can be predicted considering the kinetic model reported in Fig. 4a. In agreement with the results of the AIMS simulations, we assume that the cis-trans isomerization and the DHP cyclization happen on the excited state whereas the helical inversion process takes place only on $\mathrm{S}_{0}$. At the photo-stationary state, the enantiomeric excess of DHP can be expressed as follows (the complete derivation is discussed in the $\mathbf{S I}$ ):

$$
e e=\frac{|[S, S D H P]-[R, R D H P]|}{[S, S D H P]+[R, R D H P]}=\begin{gathered}
\left|k_{c i s \rightarrow \operatorname{trnns}}\left(g_{D H P}-g_{\text {sti }} g_{D H P}\right)+2 k_{r a x}\left(g_{D H P}-g_{s t i l}\right)\right| \\
k_{\text {cis } \rightarrow \text { trans }}\left(1-g_{\text {sti }}\right)+2 k_{r a x}\left(1-g_{\text {sti }} g_{D H P}\right)
\end{gathered}
$$

where $g_{\text {stil }}$ and $g_{\mathrm{DHP}}$ are the $g$ factors of cis-stilbene and DHP, respectively (Fig. S2), while $k_{\text {cis@trans }}$ and $k_{\text {rac }}$ represent the kinetic constants of the cis-trans photoisomerization reaction, and the ground state helical inversion process, respectively. The above expression demonstrates how the ee depends on the asymmetry of the electronic excitations (via the $g$ factors), the cis-trans photoisomerization and the ground state helical inversion processes.

For a given set of $g$ factors, three limiting cases arise: the cis-trans photoisomerization is faster than the ground state helical inversion $\left(k_{\text {cis@trans }} \gg k_{\text {rac }}\right.$ ), $\mathrm{S}_{0}$ racemization is faster than the cis-trans isomerization $\left(k_{\text {rac }} \gg k_{\text {cis@trans }}\right)$ or these processes take place on comparable time scale ( $\left.k_{\text {cis@trans }} \sim k_{\text {rac }}\right)$. The wavelength-dependent enantiomeric excess of DHP is reported for the three cases in Fig. $\mathbf{4 b}$. According to our simulation, the maximum enantiomeric excess reached around $250 \mathrm{~nm}$ is $\sim 0.23 \%$ when the photoisomerization is faster than the helical inversion process (which is the case for cis-stilbene due to its sub-picosecond cis-trans photoisomerization $\left.{ }^{10-15}\right)$, whereas it is $\sim 0.25 \%$ in the other two limit cases. The predicted ee is comparable with what Butchardt and co-workers reported for the asymmetric synthesis of chiral helicenes with CPL. ${ }^{23-25}$ In these early experiments, the different CPL absorption of the enantiomeric conformations of the diarylethylene substrate led to an optical yield of about $0.2 \% .{ }^{24,25}$ More recently, Feringa and co-workers were able to induce asymmetric photoisomerization in sterically overcrowded alkenes where they reached an ee of $0.07 \% 26-28$ by enantioselectively exciting a racemic mixture of thermally stable enantiomers (free enthalpy of racemization larger than $20 \mathrm{kcal} / \mathrm{mol}$ ) with CPL leading to unidirectional photo-isomerization. 
Our kinetic model shows that the expected enantiomeric excess is strongly dependent on the $g$ factors. Considering that the anisotropy factors are typically quite small (less than 0.01) a low ee should be expected. ${ }^{23}$ Future improvements may include the excitation to states with larger $g$ factors as well as shifting of the photo-stationary equilibrium, e.g., by selective removal of one of the enantiomeric photoproducts. Indeed, the optimal condition to maximize the ee is reached when one enantiomer is preferentially formed and the other destroyed by the same monochromatic CPL irradiation (corresponding to $g$ factors with opposite sign, see Fig. S3 for a generic A $\AA$ B photoreaction). Since the anisotropy factors are wavelength-dependent, the aim is to find the optical window that maximizes the excitation asymmetry.

In conclusion, our non-adiabatic simulations of stilbene and stiff-stilbene suggest that electronic excitation can change the inherent chiral behavior of molecules. We coin the term electronically prochiral to describe molecules whose enantiomer interconversion can be hindered on the excited electronic state, opening up the possibility for asymmetric photochemistry from an effectively nonchiral starting point. Exploiting such asymmetric photochemistry and its connection with unidirectional motion will represent the next step toward designing new generations of responsive smart materials.

\section{Declarations}

\section{Acknowledgments}

This work was supported by the MURI program of the Office of Naval Research (N00014-18-1-2624). Lawrence Livermore National Laboratory is operated by Lawrence Livermore National Security, LLC, for the U.S. Department of Energy, National Nuclear Security Administration under Contract DE-AC5207NA27344.

\section{Methods}

\section{Computational details}

The excited state non adiabatic dynamics of cis-stilbene and cis-stiff-stilbene were investigated with $a b$ initio multiple spawning ${ }^{19-21}$ interfaced with multiconfigurational electronic structure methods. GPU accelerated State-Averaged Complete Active Space Self-Consistent Field (SA-2-CASSCF(2,2)/6-31G*) was employed to model the photochemistry of cis-stilbene. This level of theory has been shown to provide a reliable description of the cis-stilbene photoisomerization PES. ${ }^{13,15}$ Modelling the excited state landscape of cis-stiff-stilbene has been shown to be a challenging task. ${ }^{10}$ Here, we employ the a-scaled Floating Occupation Molecular Orbital Complete Active Space Configuration Interaction (a-FOMO-CASCl) electronic structure method, ${ }^{29-31}$ which provides a balanced description of the twisting barriers on both the cis and trans side. The main idea behind a-FOMO-CASCl is to recover effects on the electronic state splitting arising from dynamic electron correlation that is mostly absent in the FOMO-CASCI wavefunction through an a scaling of the state-specific energy splitting while leaving the state-average 
energy untouched in the same way as a-CASSCF. ${ }^{31}$ Adopting a(0.8)-FOMO(b=0.2)-CAS(2,2) Cl/6-31G* (where $a$ is the scaling factor, and $b$ represents the FON temperature) with two electrons into two $p$ orbitals, the computed ratio of the cis and trans barriers shows good agreement with experimental estimates. Critical points of the ground and excited state potential energy surfaces are reported for cisstiff-stilbene in Fig. S4. The TeraChem electronic structure package $\mathrm{e}^{16-18}$ was employed to perform all the electronic structure calculations.

\section{Initial Conditions Generation}

Thirty initial conditions (ICs) for each enantiomer ( $P$ and $M$ ) of cis-stilbene and cis-stiff-stilbene were selected out of 500 geometries sampled from a $0 \mathrm{~K}$ harmonic Wigner distribution corresponding to geometry and frequencies computed at B3LYP/6-31G* and B3LYP/6-31G** for cis-stilbene and cis-stiffstilbene, respectively. These 500 phase space points were used to simulate the electronic absorption spectra (Fig. S5). The absorption spectra were generated by single point energy calculations at SA-2$\operatorname{CASSCF}(2,2) / 6-31 \mathrm{G}^{*}$ and $\mathrm{a}(0.8)-\mathrm{FOMO}(\mathrm{b}=0.2)-\mathrm{CAS}(2,2) \mathrm{Cl} / 6-31 \mathrm{G}^{*}$, for cis-stilbene and cis-stiff-stilbene, respectively. The final spectra were obtained by broadening the $S_{0}{ }^{\circledR} S_{1}$ excitation energies with Gaussian functions (full width half maximum of $0.2 \mathrm{eV}$ ). We randomly selected 30 different ICs from the 500 phase space points used to simulate the spectra. These ICs (positions and momenta) were placed on the $\mathrm{S}_{1}$ surface and propagated with AIMS.

\section{P-M thermal barriers}

Transition states (TS) for the $P$-M helical inversion were evaluated by the Transition State finder in DLFIND through the ChemShell/TeraChem interface. B3LYP-D3/def2-TZVP(-ff ${ }^{32-34}$ was employed. The TS involved in the helical inversion are reported in Fig. $\mathbf{S 6 .}$

\section{Simulation of Circular Dichroism spectra}

The electronic absorption and ECD spectra (Fig. S7) were computed by means of the simplified timedependent density functional theory (sTD-DFT). ${ }^{35,36}$ We used 1000 structures from the aforementioned OK harmonic Wigner distribution. At each of these structures, we computed Kohn-Sham density functional theory single-point energies with BHLYP/def2-TZVP(-f) ${ }^{34,37-39}$ using the TeraChem program. The molecular orbitals and orbital energies were then used to compute all vertical excitations up to $10 \mathrm{eV}$ with the sTD-DFT method as implemented in the sTDA program (v1.6). ${ }^{40}$ All excitations were blue-shifted by $0.7 \mathrm{eV}$ and convolved with Gaussians of $0.24 \mathrm{eV}$ width at 1/e maximum to match the previously reported experimental absorption (in hexane). ${ }^{41}$ The ECD spectra and the relative r-CPL absorption spectra were obtained and shifted accordingly. The spectroscopic data for stiff-stilbene was determined in the same way. A blue-shift of $0.35 \mathrm{eV}$ was used to match the absorption spectrum from Ref. 41.

\section{References}


1. Kottas, G. S., Clarke, L. I., Horinek, D. \& Michl, J. Artificial Molecular Rotors. Chem. Rev. 105, $1281-$ 1376, (2005).

2. Feringa, B. L. The Art of Building Small: From Molecular Switches to Molecular Motors. J. Org. Chem. 72, 6635-6652, (2007).

3. Baroncini, M., Silvi, S. \& Credi, A. Photo- and Redox-Driven Artificial Molecular Motors. Chem. Rev., (2019).

4. Koumura, N., Zijlstra, R. W. J., van Delden, R. A., Harada, N. \& Feringa, B. L. Light-driven monodirectional molecular rotor. Nature 401, 152-155, (1999).

5. Roke, D., Wezenberg, S. J. \& Feringa, B. L. Molecular rotary motors: Unidirectional motion around double bonds. Proc. Natl. Acad. Sci. 115, 9423, (2018).

6. Marchand, G., Eng, J., Schapiro, I., Valentini, A., Frutos, L. M., Pieri, E., Olivucci, M., Léonard, J. \& Gindensperger, E. Directionality of Double-Bond Photoisomerization Dynamics Induced by a Single Stereogenic Center. J. Phys. Chem. Lett. 6, 599-604, (2015).

7. Wang, J., Oruganti, B. \& Durbeej, B. Light-driven rotary molecular motors without point chirality: a minimal design. Phys. Chem. Chem. Phys. 19, 6952-6956, (2017).

8. Huber, L. A., Hoffmann, K., Thumser, S., Böcher, N., Mayer, P. \& Dube, H. Direct Observation of Hemithioindigo-Motor Unidirectionality. Angew. Chem. Int. Ed. 56, 14536-14539, (2017).

9. Guentner, M., Schildhauer, M., Thumser, S., Mayer, P., Stephenson, D., Mayer, P. J. \& Dube, H. Sunlightpowered kHz rotation of a hemithioindigo-based molecular motor. Nat. Comm. 6, 8406, (2015).

10. Quick, M., Berndt, F., Dobryakov, A. L., loffe, I. N., Granovsky, A. A., Knie, C., Mahrwald, R., Lenoir, D., Ernsting, N. P. \& Kovalenko, S. A. Photoisomerization Dynamics of Stiff-Stilbene in Solution. J. Phys. Chem. B 118, 1389-1402, (2014).

11. Fuß, W., Kosmidis, C., Schmid, W. E. \& Trushin, S. A. The Photochemical cis-trans Isomerization of Free Stilbene Molecules Follows a Hula-Twist Pathway. Angew. Chem. Int. Ed. 43, 4178-4182, (2004).

12. Liu, F. \& Morokuma, K. Computational Study on the Working Mechanism of a Stilbene Light-Driven Molecular Rotary Motor: Sloped Minimal Energy Path and Unidirectional Nonadiabatic Photoisomerization. J. Am. Chem. Soc. 134, 4864-4876, (2012).

13. Quenneville, J. \& Martínez, T. J. Ab Initio Study of Cis-Trans Photoisomerization in Stilbene and Ethylene. J. Phys. Chem. A 107, 829-837, (2003).

14. Improta, R. \& Santoro, F. Excited-State Behavior of trans and cis Isomers of Stilbene and Stiff Stilbene: A TD-DFT Study. J. Phys. Chem. A 109, 10058-10067, (2005).

15. Weir, H., Williams, M., Parrish, R. M., Hohenstein, E. G. \& Martínez, T. J. Nonadiabatic Dynamics of Photoexcited cis-Stilbene Using Ab Initio Multiple Spawning. J. Phys. Chem. B 124, 5476-5487, (2020).

16. Ufimtsev, I. S. \& Martinez, T. J. Quantum Chemistry on Graphical Processing Units. 2. Direct SelfConsistent-Field Implementation. J. Chem. Theory Comput. 5, 1004-1015, (2009). 
17. Ufimtsev, I. S. \& Martinez, T. J. Quantum chemistry on graphical processing units. 3. Analytical energy gradients, geometry optimization, and first principles molecular dynamics. J. Chem. Theory Comput. 5, 2619-2628, (2009).

18. Ufimtsev, I. S. \& Martínez, T. J. Quantum Chemistry on Graphical Processing Units. 1. Strategies for Two-Electron Integral Evaluation. J. Chem. Theory Comput. 4, 222-231, (2008).

19. Ben-Nun, M. \& Martínez, T. J. Ab initio quantum molecular dynamics. Adv. Chem. Phys. 121, 439512, (2002).

20. Ben-Nun, M. \& Martínez, T. J. Nonadiabatic molecular dynamics: Validation of the multiple spawning method for a multidimensional problem. J. Chem. Phys. 108, 7244-7257, (1998).

21. Ben-Nun, M., Quenneville, J. \& Martínez, T. J. Ab Initio Multiple Spawning: Photochemistry from First Principles Quantum Molecular Dynamics. J. Phys. Chem. A 104, 5161-5175, (2000).

22. Kuhn, W. The physical significance of optical rotatory power. Trans. Faraday Soc. 26, 293-308, (1930).

23. Rau, H. Asymmetric photochemistry in solution. Chem. Rev. 83, 535-547, (1983).

24. Bernstein, W. J., Calvin, M. \& Buchardt, O. Absolute asymmetric synthesis. I. Mechanism of the photochemical synthesis of nonracemic helicenes with circularly polarized light. Wavelength dependence of the optical yield of octahelicene. J. Am. Chem. Soc. 94, 494-498, (1972).

25. Shen, Y. \& Chen, C.-F. Helicenes: Synthesis and Applications. Chem. Rev. 112, 1463-1535, (2012).

26. Feringa, B. L. In Control of Motion: From Molecular Switches to Molecular Motors. Acc. Chem. Res. 34, 504-513, (2001).

27. Feringa, B. L., van Delden, R. A., Koumura, N. \& Geertsema, E. M. Chiroptical Molecular Switches. Chem. Rev. 100, 1789-1816, (2000).

28. Huck, N. P. M., Jager, W. F., de Lange, B. \& Feringa, B. L. Dynamic Control and Amplification of Molecular Chirality by Circular Polarized Light. Science 273, 1686, (1996).

29. Slavíček, P. \& Martínez, T. J. Ab initio floating occupation molecular orbital-complete active space configuration interaction: An efficient approximation to CASSCF. J. Chem. Phys. 132, 234102, (2010).

30. Hollas, D., Šištík, L., Hohenstein, E. G., Martínez, T. J. \& Slavíček, P. Nonadiabatic Ab Initio Molecular Dynamics with the Floating Occupation Molecular Orbital-Complete Active Space Configuration Interaction Method. J. Chem. Theory Comput. 14, 339-350, (2018).

31. Snyder, J. W., Parrish, R. M. \& Martínez, T. J. a-CASSCF: An Efficient, Empirical Correction for SACASSCF To Closely Approximate MS-CASPT2 Potential Energy Surfaces. J. Phys. Chem. Lett. 8, 2432-2437, (2017).

32. Becke, A. D. Density-functional thermochemistry. III. The role of exact exchange. J. Chem. Phys. 98, 5648-5652, (1993).

33. Grimme, S., Antony, J., Ehrlich, S. \& Krieg, H. A consistent and accurate ab initio parametrization of density functional dispersion correction (DFT-D) for the 94 elements H-Pu. J. Chem. Phys. 132, 154104, (2010). 
34. Weigend, F. \& Ahlrichs, R. Balanced basis sets of split valence, triple zeta valence and quadruple zeta valence quality for $\mathrm{H}$ to $\mathrm{Rn}$ : Design and assessment of accuracy. Phys. Chem. Chem. Phys. 7, 32973305, (2005).

35. Bannwarth, C. \& Grimme, S. A simplified time-dependent density functional theory approach for electronic ultraviolet and circular dichroism spectra of very large molecules. Comp. Theo. Chem. 1040-1041, 45-53, (2014).

36. Grimme, S. A simplified Tamm-Dancoff density functional approach for the electronic excitation spectra of very large molecules. J. Chem. Phys. 138, 244104, (2013).

37. Becke, A. D. Density-functional exchange-energy approximation with correct asymptotic behavior. Phys. Rev. A 38, 3098-3100, (1988).

38. Lee, C., Yang, W. \& Parr, R. G. Development of the Colle-Salvetti correlation-energy formula into a functional of the electron density. Phys. Rev. B 37, 785-789, (1988).

39. Becke, A. D. A new mixing of Hartree-Fock and local density-functional theories. J. Chem. Phys. 98, 1372-1377, (1993).

40. <https://github.com/grimme-lab/stda> (

41. Taniguchi, M. \& Lindsey, J. S. Database of Absorption and Fluorescence Spectra of $>300$ Common Compounds for use in PhotochemCAD. Photochem. Photobiol. 94, 290-327, (2018).

\section{Figures}

a)

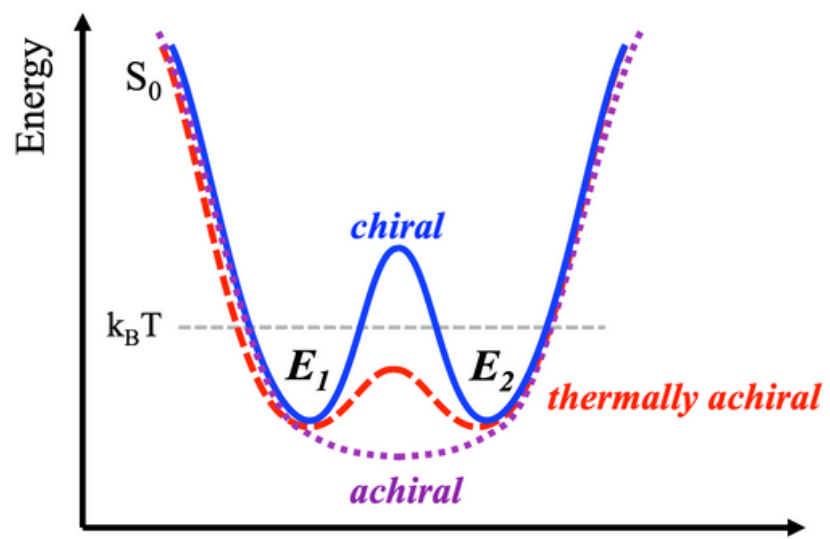

$\{\mathrm{R}\}$

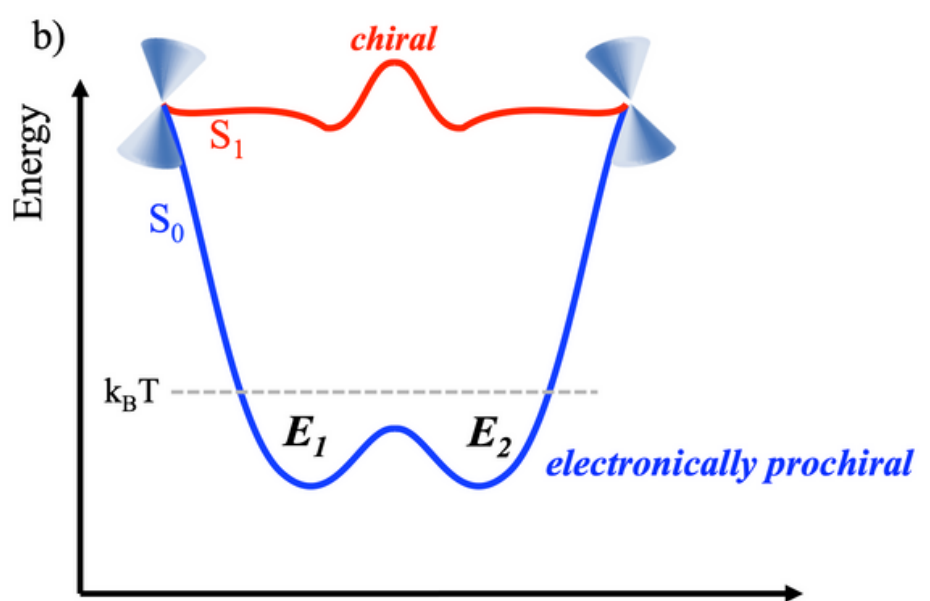

$\{\mathrm{R}\}$ 


\section{Figure 1}

a) Potential Energy Surfaces (PESs) for chiral and achiral molecules. A barrier separates two minima corresponding to the two enantiomers (E1 and E2): if the interconversion barrier is much higher than the thermal energy (kBT), the molecule is chiral, otherwise rapid interconversion between the two enantiomeric configurations takes place making them inseparable (thermally achiral molecules). b) PESs for electronic prochiral molecules which can be converted from achiral to chiral by electronic excitation. The example here shows a case where the molecule is thermally achiral on the ground state, but a similar scenario is also possible where the molecule is truly achiral on the ground state.

a)

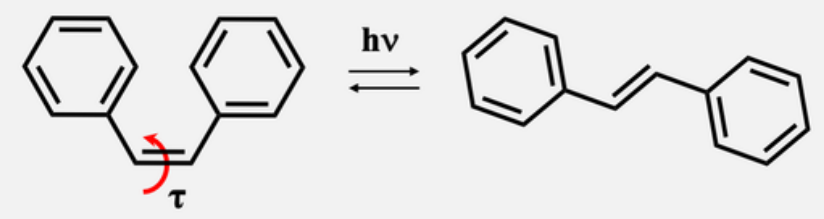

cis-stilbene

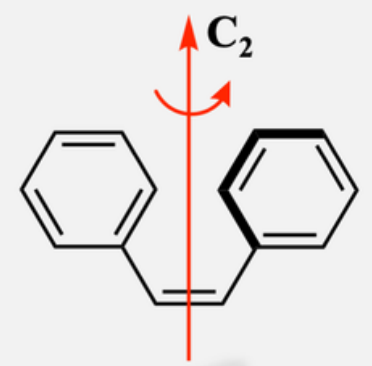

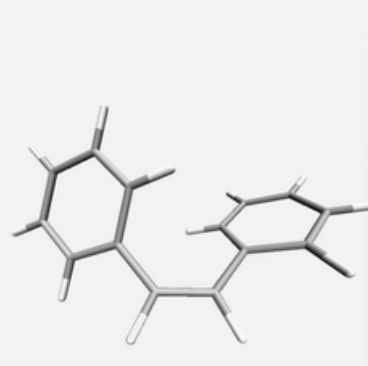

$\boldsymbol{M}$

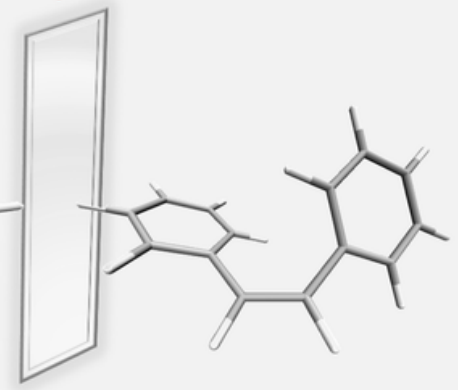

$\boldsymbol{P}$ b)

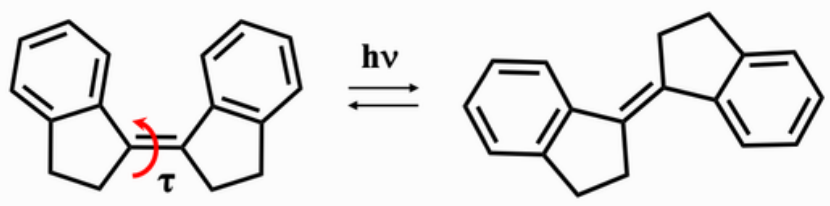

cis-stiff-stilbene
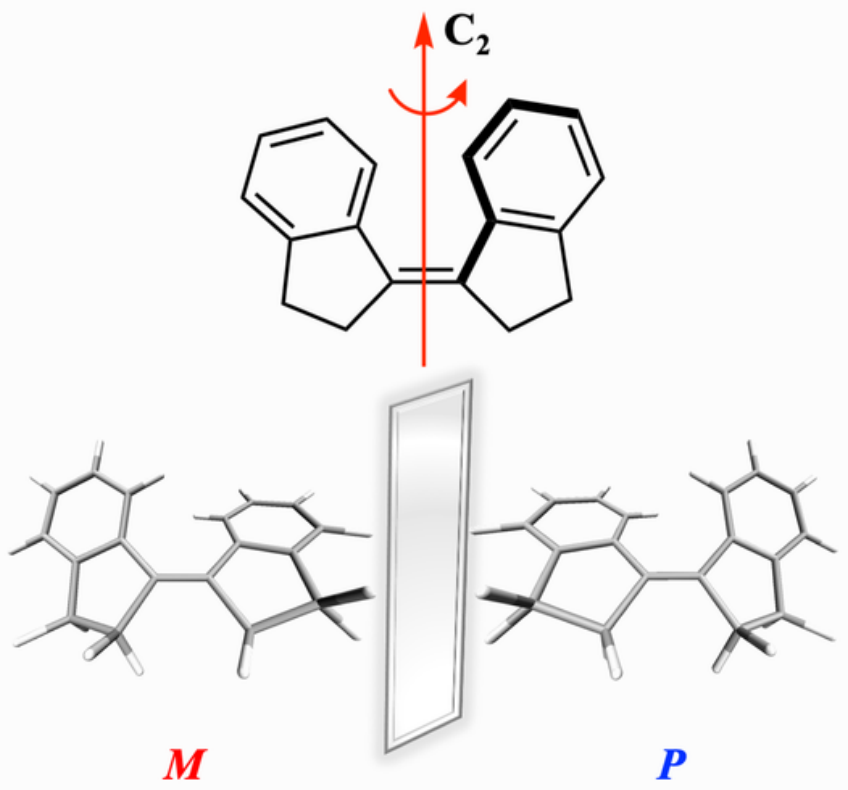

\section{Figure 2}

$\mathrm{P}$ and $\mathrm{M}$ enantiomers of cis-stilbene a) and cis-stiff-stilbene $\mathrm{b}$ ). The $\mathrm{C} 2$ rotational axis is highlighted in red along with the twist angle $(\tau)$ around the carbon-carbon double bond (defined as the average of the two dihedral angles involving the central $\mathrm{C}=\mathrm{C}$ double bond). 
a)

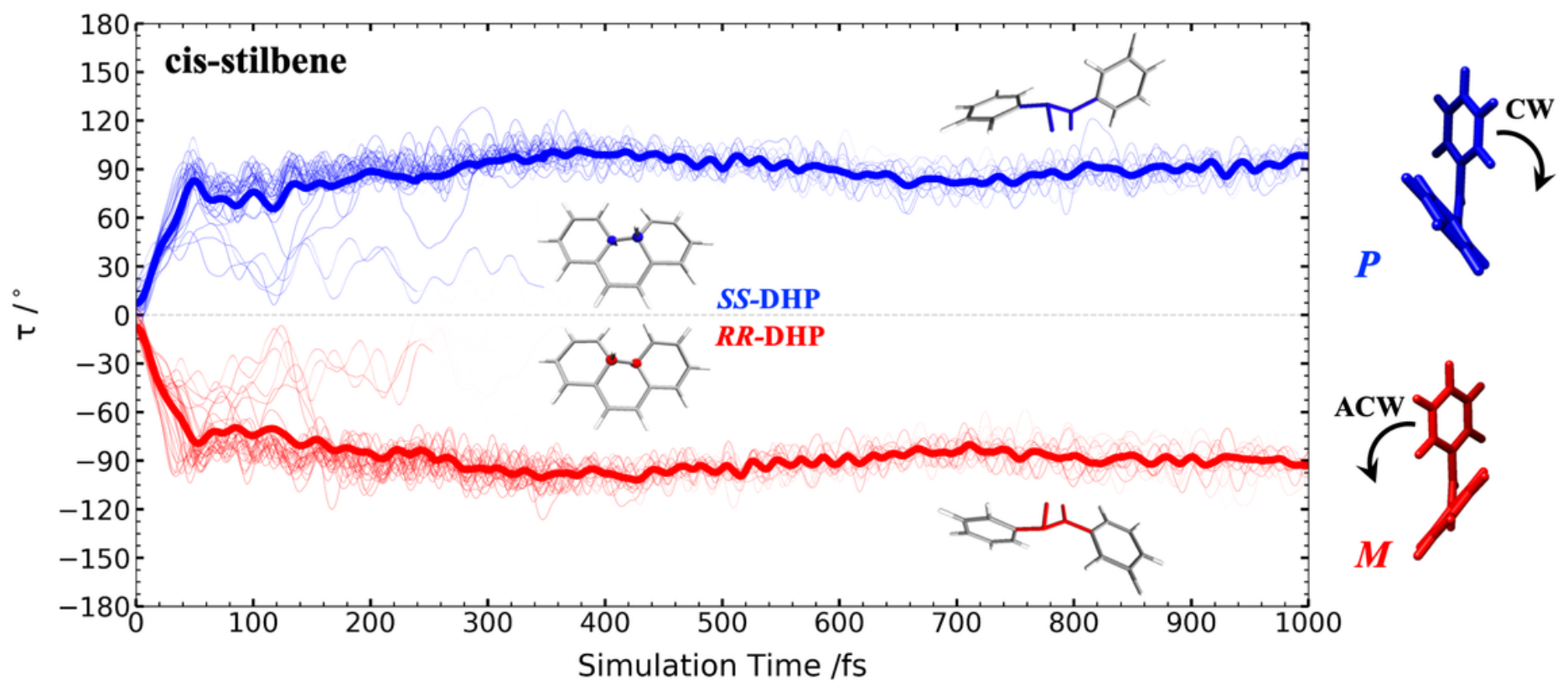

b)

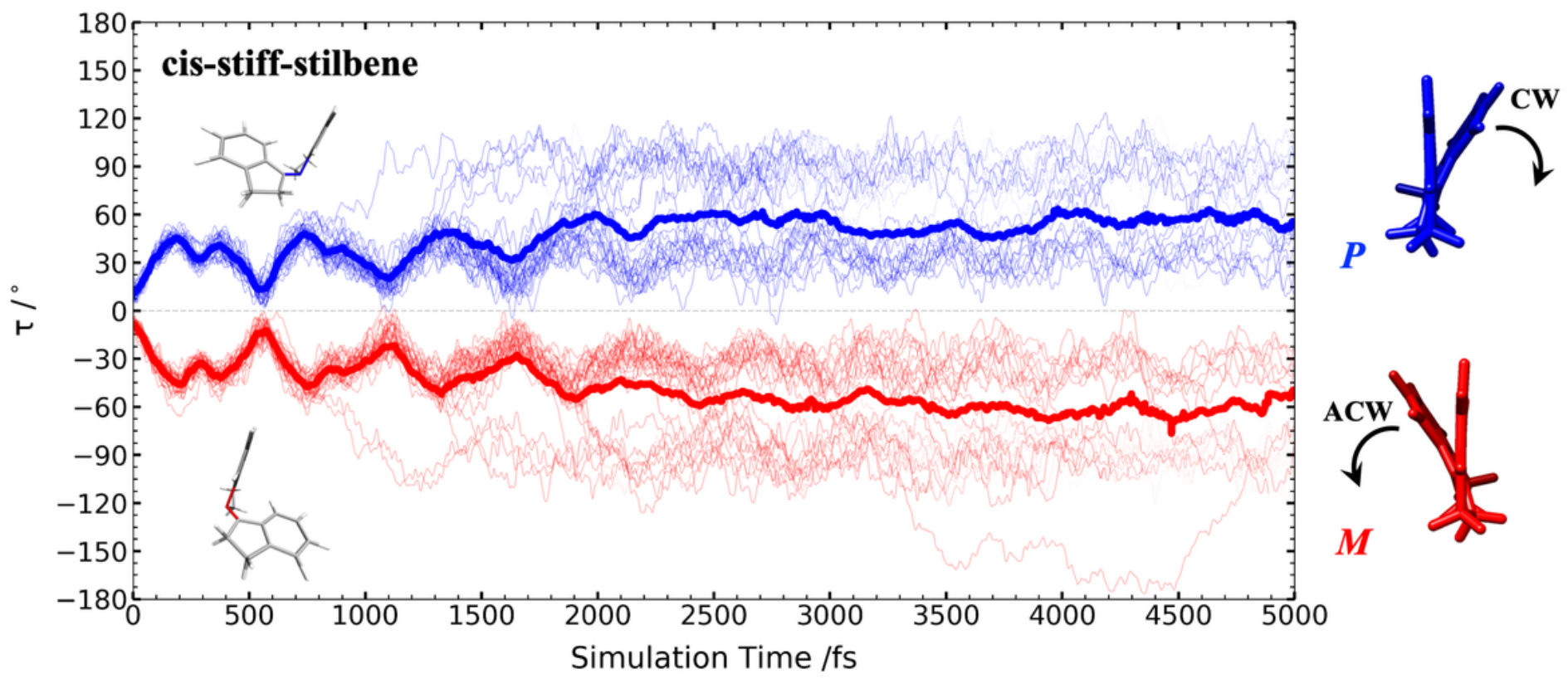

\section{Figure 3}

Time evolution of the, $\tau$, twist angle on the $\mathrm{S} 1$ state for the two enantiomers ( $\mathrm{P}$ blue and $\mathrm{M}$ red) of cisstilbene a) and cis-stiff-stilbene b). The bold line represents the weighted average of the total population over all the trajectory basis function (TBFs) on S1. Each line represents a TBF on S1, where its thickness of each line is proportional to its contribution to the weighted average. 


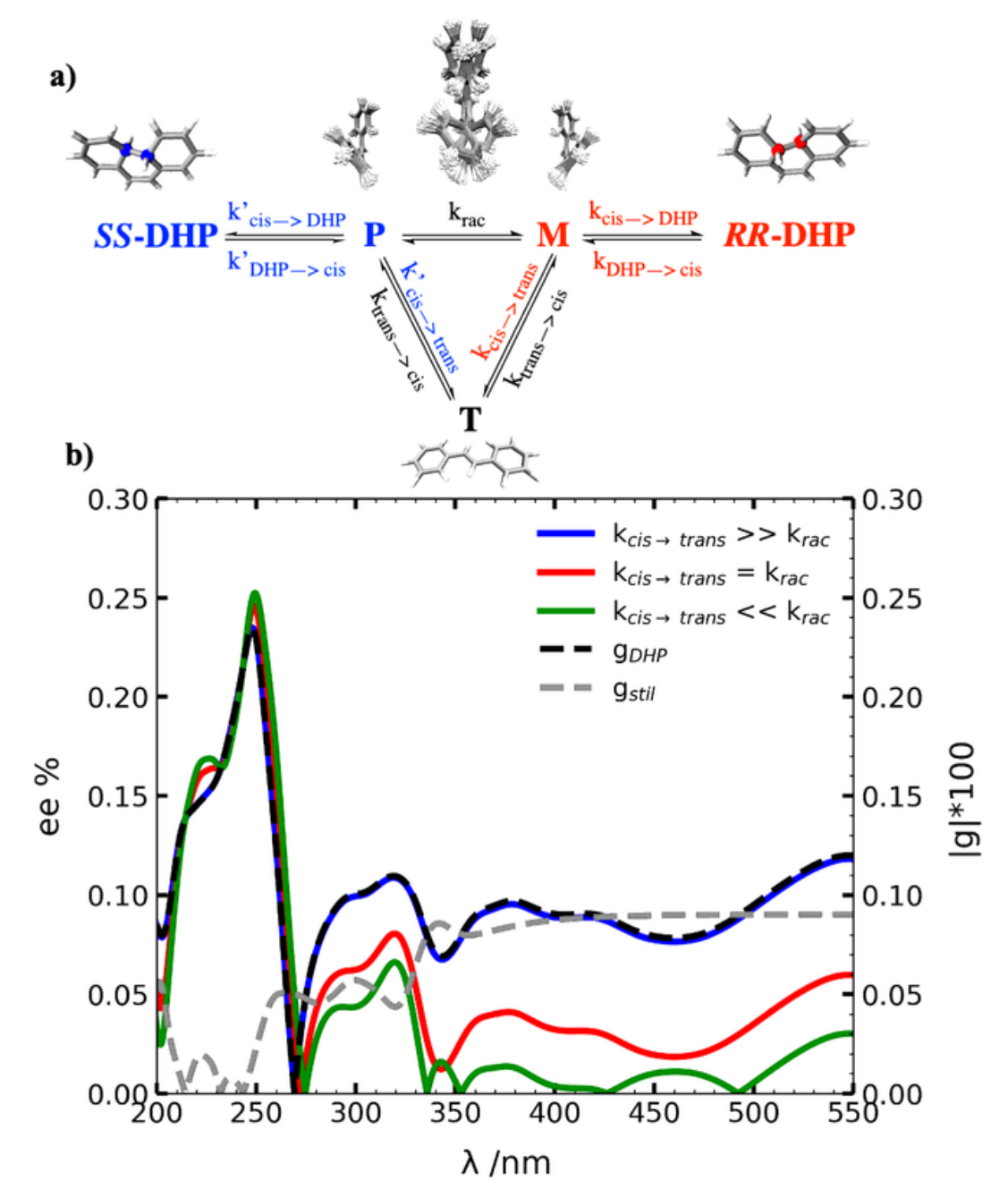

Figure 4

a) Kinetic model employed to describe the photochemistry of cis-stilbene. In agreement with the results of the AIMS dynamics, we are assuming that the cis-trans isomerization and the DHP cyclization happen on the excited state whereas the helical inversion process takes place only on SO. Thus, the excitation of the $\mathrm{M}$ and $\mathrm{P}$ enantiomers can lead to photocyclization to R,R-DHP or SS-DHP with kinetic constants kcis $\rightarrow$ DHP and k'cis $\rightarrow$ DHP or photoisomerization to the achiral trans-stilbene $(T)$ with kinetic constants $\mathrm{kcis} \rightarrow$ trans and $\mathrm{k}^{\prime} \mathrm{cis} \rightarrow$ trans, respectively. At the same wavelength, the DHP enantiomers can be excited back leading to the $\mathrm{M}$ and $\mathrm{P}$ enantiomers with kinetic constants $\mathrm{kDHP} \rightarrow$ cis and $\mathrm{k}^{\prime} \mathrm{DHP} \rightarrow$ cis. transStilbene can also be excited, producing both $\mathrm{P}$ and $\mathrm{M}$ cis-stilbene conformations (ktrans $\rightarrow$ cis). $\mathrm{P}$ and $\mathrm{M}$ are considered to be in equilibrium in the ground state with a helical inversion constant krac. $b$ ) Enantiomeric excess reached at the photo-stationary state exciting a racemic mixture of the $\mathrm{P}$ and $\mathrm{M}$ enantiomers of cis-stilbene with r-CPL as a function of the excitation wavelength. Three limit cases were considered according to eq 1. The wavelength-dependent $\mathrm{g}$ factors for stilbene (gstil) and DHP (gDHP) are also reported as dashed lines. In the optical window 250-350 nm, the $M$ enantiomer preferentially absorbs the r-CPL leading to trans-stilbene and R,R-DHP (Fig. S2a). This latter is also preferentially excited by the r-CPL retrieving cis-stilbene and resulting in an overall optical enrichment of S,S-DHP. On the other hand, in the optical window 200-270 nm (Fig. S2b), the band in the DHP ECD spectrum changes 
sign and the r-CPL is preferentially absorbed by the S,S-DHP enantiomer (i.e., the anisotropy factor $\mathrm{g}$ changes sign), resulting into an excess of R,R-DHP.

\section{Supplementary Files}

This is a list of supplementary files associated with this preprint. Click to download.

- Supplemental.pdf 